PNL-SA- 18537

PNL-SA- -18537

COINCIDENCE MEASUREMENTS OF

DE91 005677

ELECTRON CAPTURE AND LOSS IN

ION-ATOM COLLISIONS

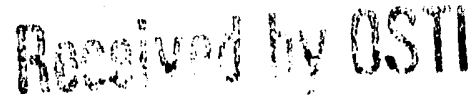

R. D. DuBois

JAN 041931

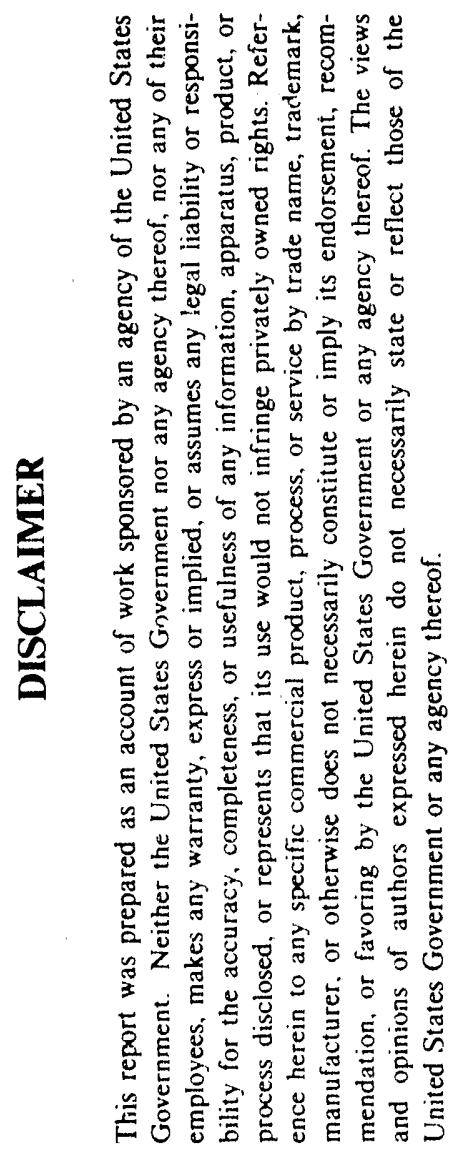

September 1990

Prepared for

4 th Workshop on High-Energy

Ion-Atom Collision Processes

Debrecen, Hungary

September 17-19, 1990

Work supported by the Office of

Health and Environmental Research

(OHER), U. S. Department of Energy

under Contract DE-ACO6-76RLO 1830

Work supported by the Office of

Health and Environmental Research

(OHER), U. S. Department of Energy

under Contract DE-ACO6-76RLO 1830

Pacific Northwest Laboratory

Richland, Washington 99352 


\title{
Coincidence Measurements of Electron Capture and Loss in Ion-Atom Collisions
}

\author{
R.D. DuBois \\ Pacific Northwest Laboratory \\ P.0. Box 999 \\ Richland, WA 99352 USA
}

\section{Abstract:}

Collisions between fast, fully stripped projectiles and atomic targets predominantly result in target electrons being ejected to the continuum. For fast partially stripped projectiles which bring weakly bound electrons into the collision, projectile ionization can also contribute to the observed electron spectra. At lower impact velocities, electron capture by the projectile ion becomes important and higher order processes, often referred to as transfer ionization, can be a significant source of free electrons. In recent years, coincidence techniques have been used to evaluate the relative importance of electron capture and loss in free electron production, to separate the capture and loss contributions from those resulting from target ionization alone, and to provide more detailed information about electron capture and loss mechanisms than is available from total cross section measurements. A brief survey of these experiments will be presented.

\section{Introduction:}

Fast charged particles traversing gaseous media gradually lose energy and slow down as they interact with individual target atoms. The energy iransferred to the target as a result of these interactions primarily results in ionization of a bound target electron, excitation to discrete excited states being less important. If the projectile also contains loosely bound electrons, projectile ionization can be an additional source of free electrons. Thus, studying the ejected electron spectra can provide information about the interaction and energy loss processes.

For the purpose of this paper, ionizing collisions will arbitrarily be classified as follows. 1) Fast, fully stripped ion impact where target ionization dominates, 2) lower velocity collisions involving fully, or partially, stripped ion impact where electron capture becomes important, and 3) fast collisions, involving projectile ions having loosely bound electrons of their own, where electron loss from the projectile is important. 
For category 1, numerous experimental and theoretical studies have demonstrated that the first Born approximation can adequately describe single target ionization resulting from fully stripped ion impact. Typically the theoretical predictions are poorer for heavier targets because of the increased probability for multiple electron emission and because more sophisticated target wave functions are required. In general, however, our understanding of these collisions is rather good.

Category 2, electron capture by the projectile, becomes important at lower impact velocities, i.e. Where the impact velocity is approximately equal to, or smaller than, the velocity of the bound target electron. Simple electron capture results in target ionization, but higher order processes, such as transfer ionization, can liberate additional target electron(s) to the continuum. Our present theoretical understanding of this process is much poorer than for fast, fully stripped ion impact because perturbative techniques such as the Born approximation are inappropriate at these low velocities. More sophisticated theoretical treatments are required. However these processes are attractive from a theoretical viewpoint since they involve a two electron transition with one of the final states being a discrete bound state while the other is a continuum state.

For interactions where the projectile brings loosely bound electrons of its own into the collision, category 3, electron loss tends to be important. Although the collision velocities are sufficiently large that perturbative techniques can be applied, the theoretical description must consider ionization of the target and of the projectile with, and without, simultaneous excitation of the other collision partner. Each of these processes needs to be modeled and calculated independently and then summed to yield information about the observed electron omission spectra. In addition, for both target and projectile ionization, the interaction involves a screened nuclear charge. Hence modeling these collisions and comparing with experimental data is often complicated.

Therefore, understanding collisions where electron capture or loss occur is one of the next major steps in extending our knowledge of ion-atom collisions. This requires experimental data capable of a) steering the theoretical models in the proper direction by indicating the relative importance of the various ionization processes and b) testing the models in order to identify their successes and failures. Sometimes it is possible to use total cross section or ditrerential electron emission measurements obtained using non-coincidence techniques for these purposes. However, in recent years coincidence techniques have been applied in order to investigate electron capture and loss mechanisms in greater detail. For a summary of the work done in this field prior to 1980 see reference 1.

In this paper examples of various coincidence methods will be presented in order to demonstrate what information can be derived from each. I wish to emphasize that the examples cited were chosen for illustrative purposes and I do not mean to imply that they represent the only research efforts in this field. The experiments discussed are intended to demonstrate what additional knowledge can be derived using 
these techniques and also to serve as a starting point for obtaining references and information in this field.

Because of space limitations, examples will be restricted to those pertaining to the emission of free electrons. One technique that has been applied toward this end is the study of coincidences between charge state analyzed projectile ions and either recoil ions or emitted electrons. Another technique is to study coincidences between scattered projectile ions and again either recoil ions or emitted electrons. From these measurements a better understanding of these ionizing collisions is gradually emerging.

\section{Electron Capture:}

As previously stated, for projectile velocities comparable to or less than the velocity of the bound target electrons, electron transfer from the target to the projectile becomes important. For example, total cross section measurements for $\mathrm{H}^{+}$ and $\mathrm{He}^{+}$impact on various atomic and molecular targets [2-4] have shown that the probability of electron capture can exceed the total ionization cross section (meaning the electron production cross section) at low impact velocities. When the capture cross section is compaidis? to or larger than the electron production cross section, higher order effects, such as transfer ionization, can then become relatively important in the production of free electrons.

\section{a) Projectile ion-recoil ion coincidences:}

At the Pacific Northwest Laboratory we have used the charge state analyzed projectile ion-recoil ion coincidence technique to study light ion impact on various atomic targets. [4-6] These studies showed that the relative number of free electrons produced via the electron capture process, in other words the transfer ionization process, was larger for targets possessing more loosely bound electrons, e.g. Ar, $\mathrm{Kr}$, than for those with fewer or more tightly bound electrons, e.g. He, Ne. However, even for $\mathrm{H}^{+}$and helium ion impact, transfer ionization was found to be a significant source of electrons in slow collisions. This is demonstrated in Fig. 1.

Even larger probabilities were found for heavier or more highly charged projectile ions. This can also be seen in Fig. 1 but a better example involves slow highly charged ion impact on helium [7] where transfer ionization can dominate in the production of free electrons. The Leningrad and Belfast groups have also used projectile ion-recoil ion coincidence techniques to study these processes [8-10]. 


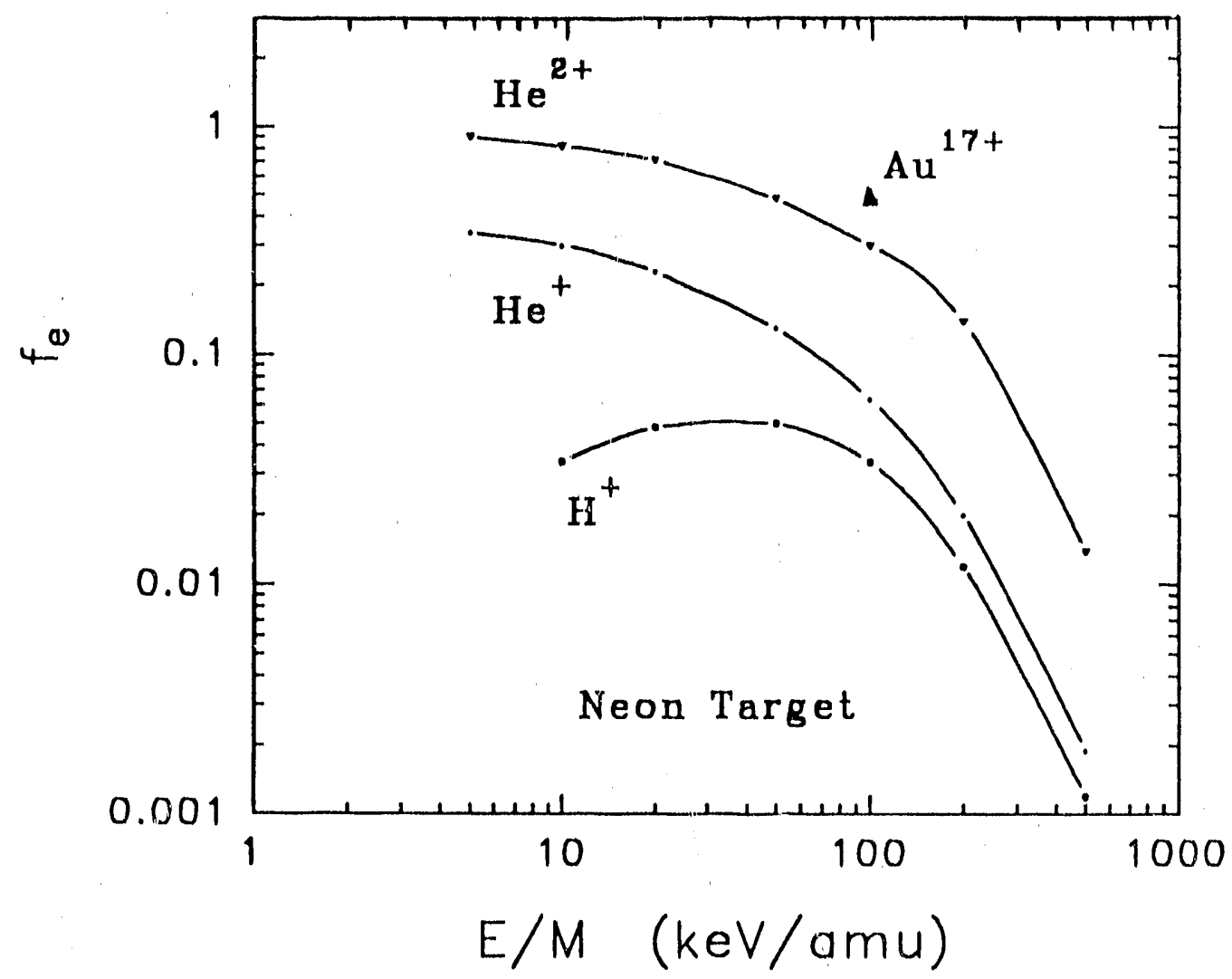

Fig. 1 The fraction of the total electron production resulting from higher order electron capture processes for viarious projectiles colliding with neon. $\mathrm{H}^{+}$impact,

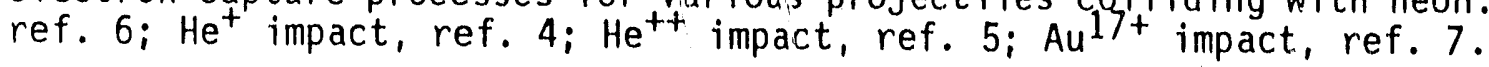

\section{b) Projectile ion-emitted electron coincidences:}

Transfer ionization can be modeled in various ways. The simplest model uses an independent particle approximation where the transfer of one target electron to the projectile is treated independently of the ionization of a second electron to the continuum, the total probability then being the product of the capture probability, $P_{C}(b)$, and the ionization probability, $P_{j}(b)$, where $b$ is the impact parameter. A second model assumes that electron-electron interactions are important. the Thomas double scattering model being a prime example. [11] In order to distinguish between these two models, differential electron spectra, measured in coincidence with charge state analyzed projectile ions, are useful.

In Fig. 2 doubly differential cross sections (DDCS) for the total electron production and for electrons originating from transfer ionization (TI) are shown. [12] Although more extensive data are required to determine the mechanism involved, these data indicate that the relative fraction of the total free electron production obtained from data as discussed in Fig. 1 is also reflected in the differential electron spectra. For example for $90^{\circ}$ electron emission in neon, the TI DDCS are roughly a factor of ten smaller than the total DDCS whereas when integrated over all emission angles and electron energies the TI contribution is roughly $5 \%$ of the total electron emission. 


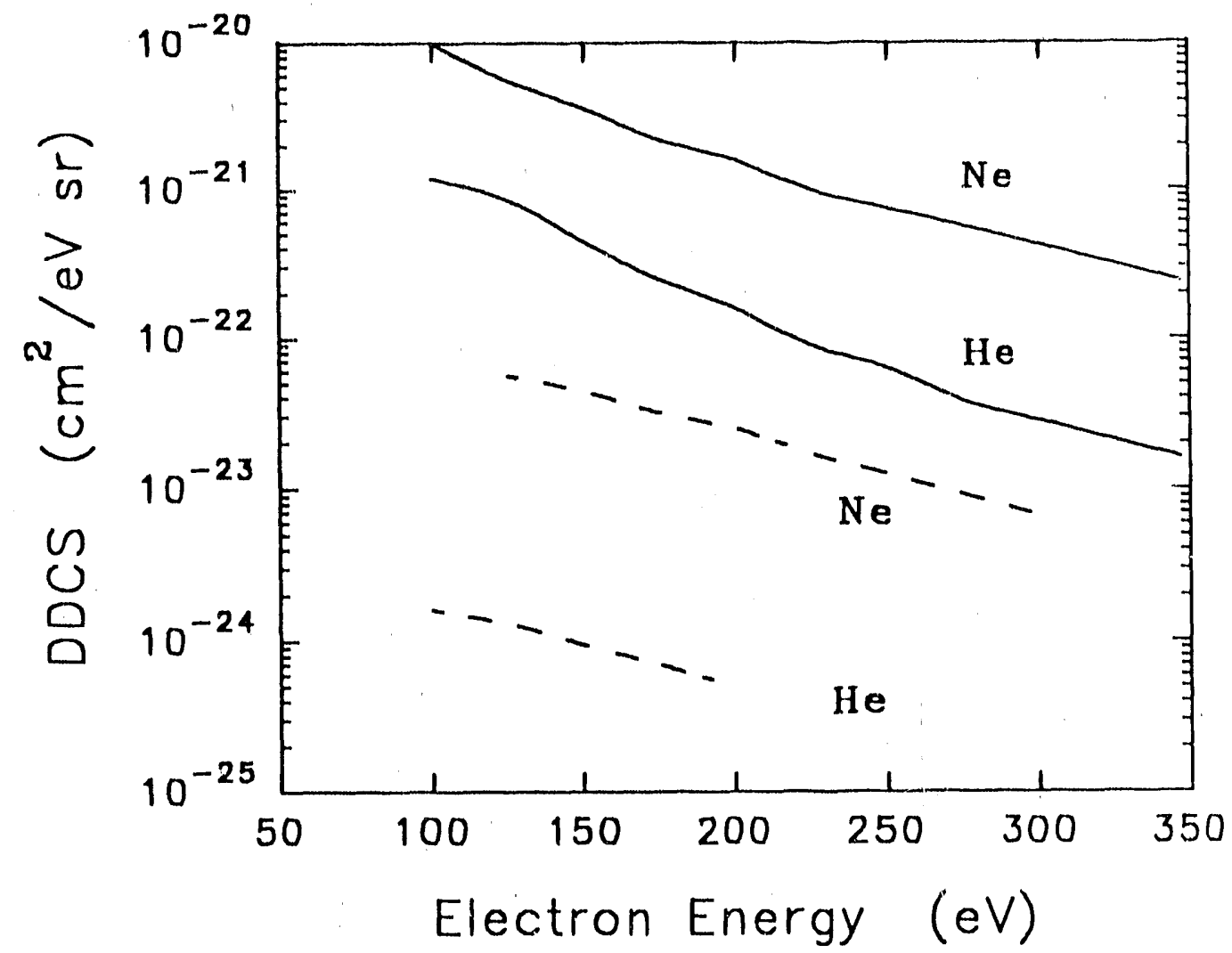

Fig. 2 Doubly differential cross sections for electron emission occurring in 300 $\mathrm{keV} \mathrm{H}^{+}$- He, Ne collisions. Solid lines, total electron emission; dashed lines, electron emission resulting from electron capturing collisions. Data are from ref. 12 .

In another experiment where coincidences between emitted electrons and projectiles that captured an electron from the target were investigated, Pálinkás et al. [13] recently demonstrated that the Thomas double scattering mechanism is responsible for the TI events occurring in fast proton-Ar collisions.

There also exists another eiectron capture mechanism which can resuit in the production of free electrons-namely electron capture to the continuum (ECC). In this process a target electron is "captured" into a continuum state of the projectile. This results in an enhancement of the number of electrons having a direction and velocity matching that of the projectile ion. The TI process can also produce electrons in the forward direction having an enhanced probability for velocities matching that of the projectile. By using charge analyzed projectile ion-zero degree emitted electron coincidence techniques, it is possible to separate these two processes from each other and from a third process that occurs when a loosely bound electron is lost from a projectile ion. This latter process, which will be discussed in a following section, is electron loss to the continuum (ELC).

As an example of the ECC, TI and ELC processes, consider $\mathrm{He}^{+}$impact. An ECC process will result in a zero degree electron - $\mathrm{He}^{+}$coincidence. TI will yield an electron in coincidence with a neutral helium atom and ELC will yield an electronionized projectile $\left(\mathrm{He}^{++}\right)$coincidence. Fig. 3 shows the relative importance of these three processes for $75 \mathrm{keV} / \mathrm{amu} \mathrm{He}$ - He collisions. These data were obtained 
in a collaborative effort between the Debrecen and Univ. Frankfurt groups. [14] In this example, transfer ionization is considerably less important than is capture to the continuum. However other studies using highly charged ions $[15,16]$ showed TI to be the dominating contributor to the cusp peak.

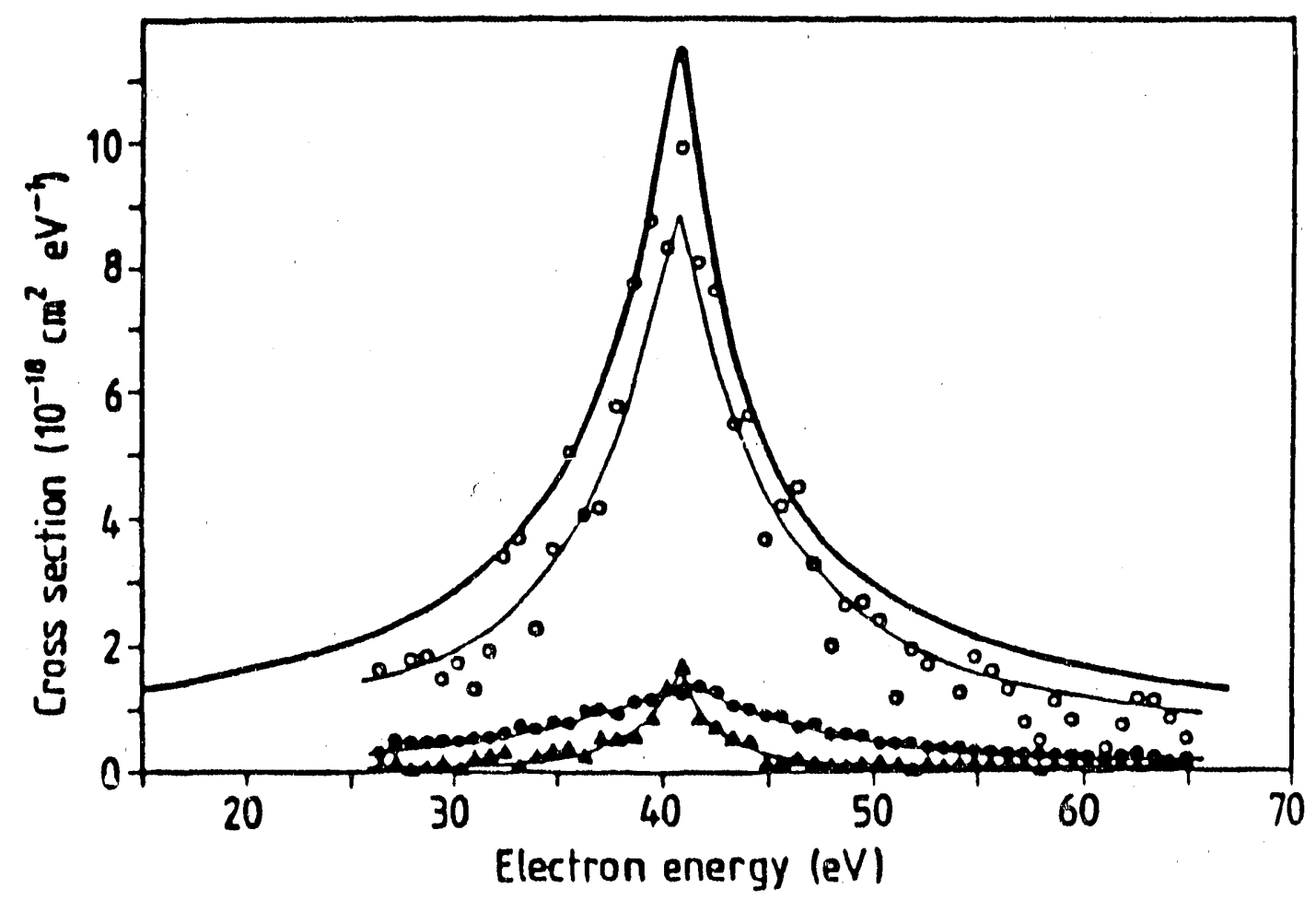

Fig. 3 Zero degree cusp electron production in $75 \mathrm{keV} / \mathrm{amu} \mathrm{He}^{+}-\mathrm{He}$ collisions indicating the relative importance of the ECC, open circles, TI, filled triangles, and ELC, filled circles, mechanisms to the total electron production, heavy solid line. Data from ref. 14.

c) Scattered projectile ion-recoil ion or emitted electron coincidences:

Another method of studying the mechanisms leading to electron emission associated with electron capture is to investigate the cross sections as a function of impact parameter. This can be done by measuming the recoil ion production as a function of the projectile ion scattering angle. According to the classical Thomas scattering mechanism, a signature of these events would be an enhancement in the differential scattering probability at $0.55 \mathrm{mrad}$ in proton-helium collisions and, in addition, the production of a doubly charged helium ion. In Aarhus, using this method a strong enhancement in the amount of doubly ionized target ions at the critical angle was found. [17] These daia, shown in Fig. 4, were interpreted as evidence of the double scattering Thomas mechanism in the TI process aithough other interpretations are possible. 


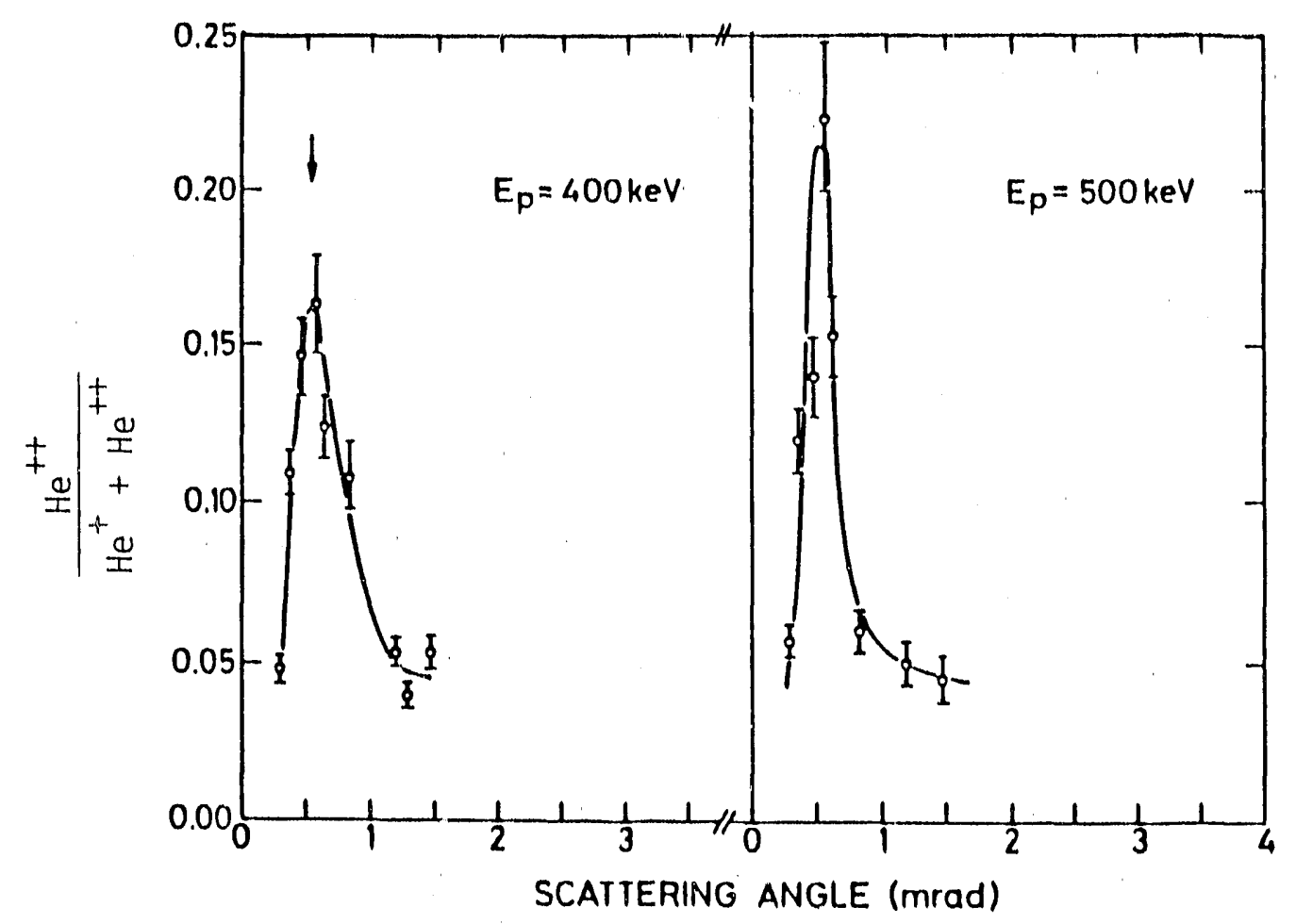

Fig. 4 Double to single ionization probabilities as a function of projectile scattering angle in $\mathrm{H}^{+}+\mathrm{He} \rightarrow \mathrm{H}+\mathrm{He}^{q^{+}}+(\mathrm{q}-1) \mathrm{e}^{-}$collisions. Data from ref. 17.

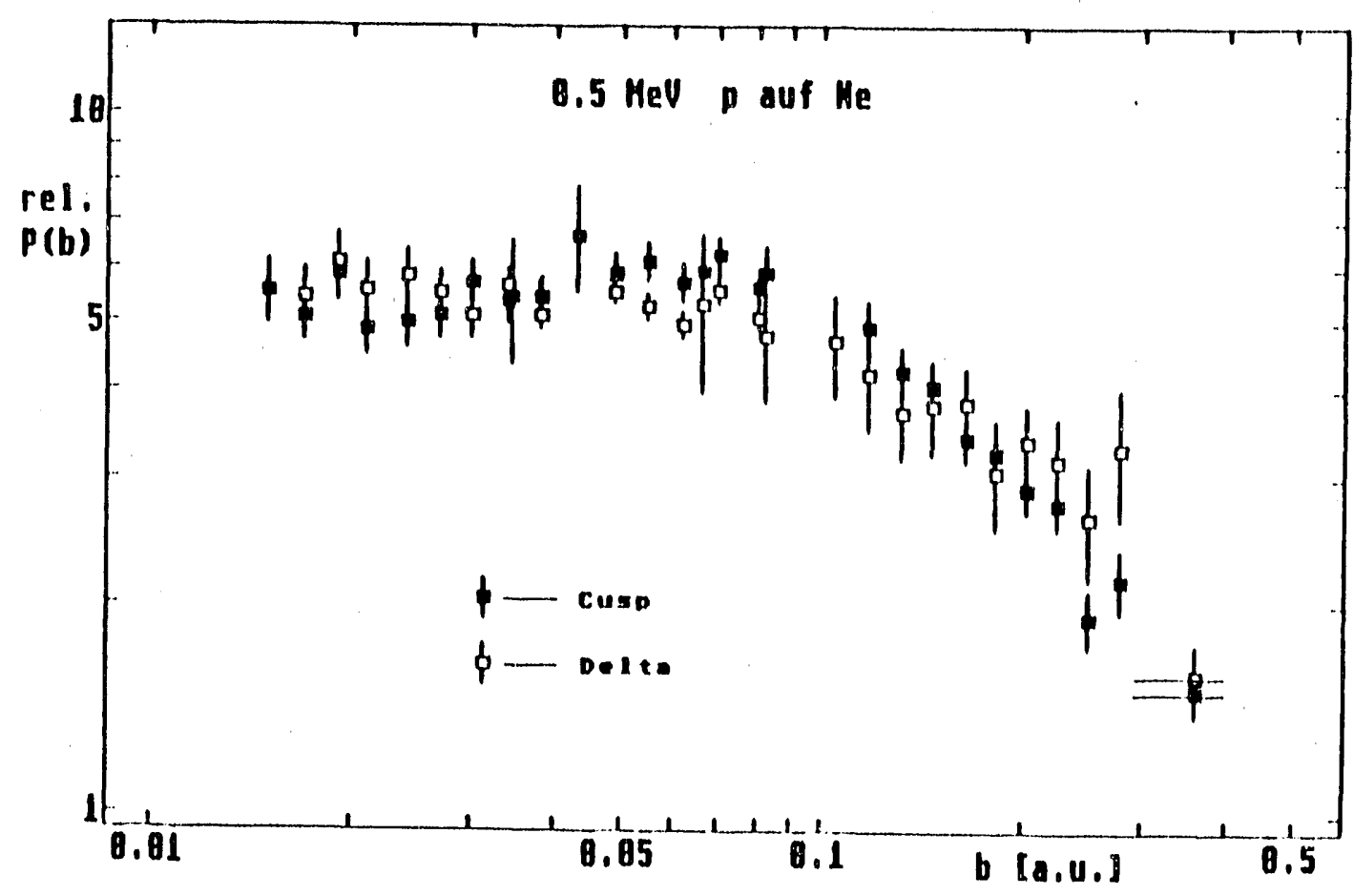

Fig. 5 Impact parameter dependencies of the cusp electron production and continuum target electrons with similar energies measured for $500 \mathrm{keV} \mathrm{H^{+ }}$ - Ne collisions. Data from ref. 19.

By measuring the scattering angles of charge state analyzed projectile ions in coincidence with zero degree electron emission, the groups at Kansas State 
University [18] and the University of Frankfurt [19] have studied the ECC and TI processes. Heavy (fluorine) and light (protons, helium ions) projectiles were used at the two laboratories respectively. For light ion impact, one of the results of this work was the demonstration that cusp electrons and continuum (delta) electrons of similar energies have identical impact parameter dependencies (see Fig. 5). This confirms that ECC cusp electrons are delta electrons that are swept in the forward direction due to the coulomb attraction of the projectile ion. From these examples, it should be clear that the use of coincidence methods has greatly contributed to our present understanding of ionization associated with electron capture processes. Certainly other interesting studies have been performed but the examples and references cited here should provide the interested reader with an overview of the field.

\section{Electron loss:}

Let us now turn our attention to collisions involving projectiles containing loosely bound electrons of their own. For these collisions an additional electron production mechanism, namely projectile ionization or electron loss to the continuum $(E L C)$, exists. In these collisions either the target, or the projectile, can be ionized and, in either case, the collision partner can remain in the ground state or be excited to a discrete or continuum state. Also in both cases the coulomb interaction involves a screened nuclear charge. Thus interpreting the observed electron spectrum is vastly more complicated than for fully stripped ion impact.

In recent years several experiments have been performed where coincidence methods have been used to separate and identify the various ionization processes occurring in these collisions. Examples of a few of these experiments are given below.

\section{a) Projectile ion-recoil ion coincidences:}

Using projectile ion-recoil ion coincidence techniques in our laboratory, we have investigated the relative importance of the electron loss channels toward to total electron production resulting from 1 ight ion impact. [4] In Fig. 6 the total electron loss cross sections along with the results of the coincidence measurements are shown for $\mathrm{He}^{+}$impact on helium and argon. The nomenclature used for the cross sections is that the number indicates the final target charge state, e.g. $1,2, \ldots$ imply that the target was singly, doubly, et cetera, ionized in a collision where the $\mathrm{He}^{+}$projectile was also ionized. Due to the coincidence technique employed, pure electron loss by the projectile cannot be investigated since it does not form a target recoil ion. However by summing the cross sections ( $\nabla)$ for simultaneous projectile-target ionization (where the number is non zero) and comparing with total 
loss cross sections obtained from a separate experiment, the pure electron loss cross section (dashed curve labeled by 0 ) can be deduced.

As can be seen in Fig. 6, for a light target such as helium roughly half of the time ionization of the projectile results in target ionization as well. For a heavier target, the sum of the coincidence cross sections equals the total loss cross section implying that pure projectile ionization is an unlikely event; collisions where projectile ionization occur always cause target ionization.

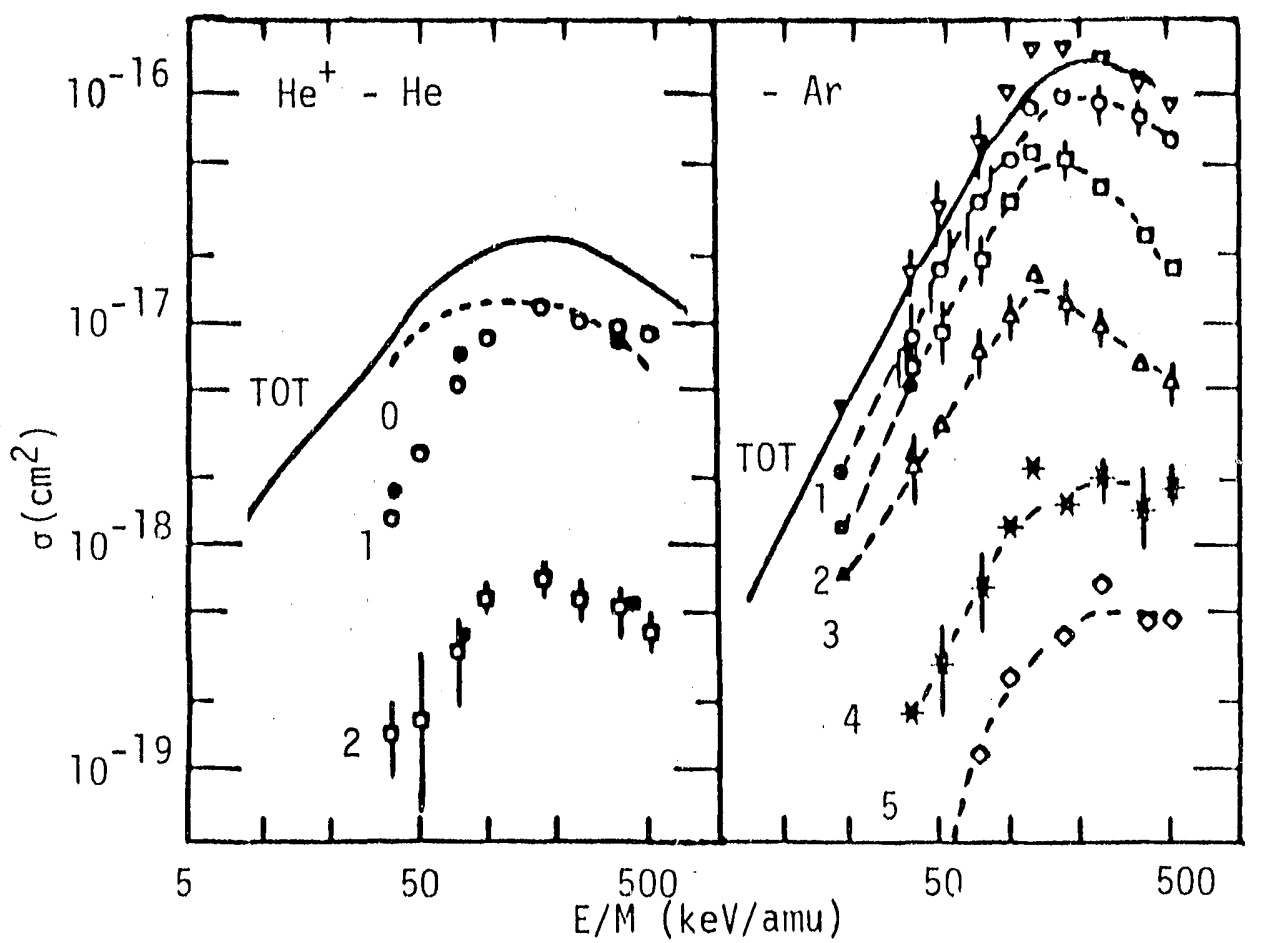

Fig. 6 Cross sections for ionization occurring in $\mathrm{He}^{+}-\mathrm{He}, \mathrm{Ar}$ collisions. Solid curves, total cross sections for electron loss by the projectile taken from refs. 3 and 20. Dashed curves though symbols, cross sections for ionization of both the projectile and the target in the collision. For argon, $\nabla$ denotes the sum of the cross sections for simultaneous projectile-target ionization. Data taken from ref. 4. The number denotes the degres of target ionization. For the helium target the curve denoted by zero is for pure electron loss by the projectile. See text for details.

Projectile ion-recoil ion coincidence data also can be used to determine the relative importance of the electron loss channels toward the tocal electron production. At higher impact energies, electron capture is relacively unimportant. Thus the total electron production comes from 1) ejecting an electron from the target, or 2) ejecting an electron from the projectile, or 3) ejecting electron from both the target and the projectile. The latter case will be called simultaneous ionization although there is no experimental or theoretical evidence implying that this is a correlated or uncorrelated process.

Examples of the relative importance of these three processes for $\mathrm{He}^{+}$impact on helium and argon are shown in Fig. 7. The contribution of projectile electrons, process 2, (designated by $P$ ) are seen to be roughly independent of the target for fast collisions. At lower impact energies there is a greater contribution for the 
lighter target because the number of loosely bound target electrons is larger for the heavier target and hence the target ionization contribution increases.

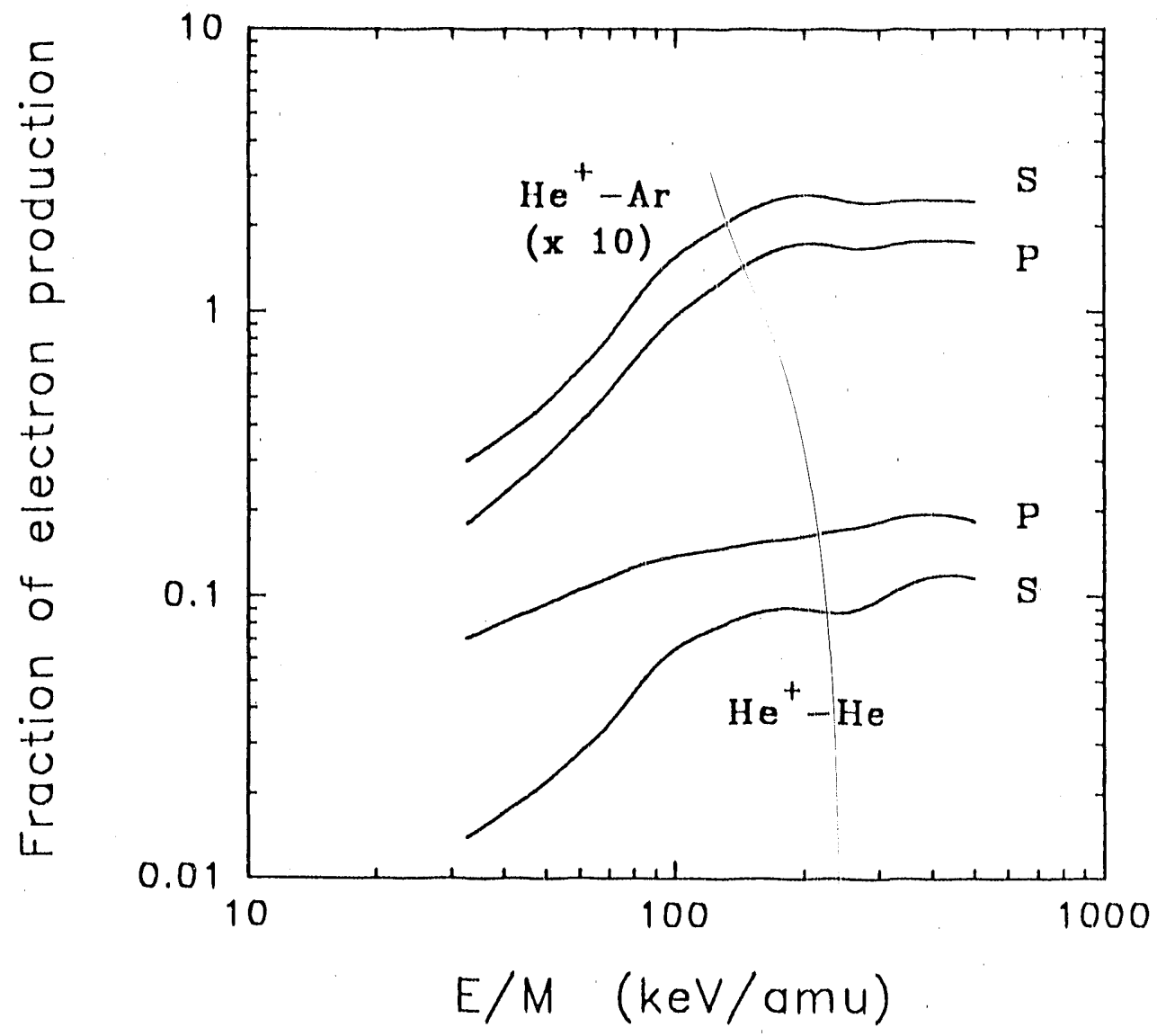

Fig. 7 The relative importance of electrons originating from the projectile (P) and those originating from the target in collisions where the projectile is also ionized (S) for He $\mathrm{H}^{+}$impact on helium and argon targets. The argon data have been shifted by a factor of 10 for display purpose's only. Data from ref. 4.

The remainder of the electrons originate from the target. However, a portion of these electrons are emitted in collisions where the projectile is also ionized, process 3 as described above. This process is of interest since a two electron transition has taken place. The contribution of these electrons (designated by $S$ in Fig. 7) is relatively important for both light and heavy targets. The combined processes, 2 and 3, indicate that, at higher impact energies a third to nearly half of the emitted electrons result from collisions where electron loss by the projectile occurs.

\section{b) Projectile ion-emitted electron coincidences:}

Additional details of the projectile and simultaneous ionization processes can be obtained by studying the differential electron emission spectra in coincidence with projectile ions that have lost an electron during the collision. These data can be used to test our theoretical urderstanding of these processes. Several years 
ago we reported data for $\mathrm{He}^{+}$- He collisions where electron emission angles of $20^{\circ}$ and $30^{\circ}$ were studied. [21] Comparisons of the experimental data with first Born calculations indicated that the theoretical treatment of target and projectile ionization were reasonably good but the theoretical model used severely underestimated simultaneous ionization events. In a recent study, [22] we extended this study to other targets. Fur an argor target, a wider range of emission angles was studied. In this case very pcor agreement between experiment and theory was found.

In order to clarify the situation, additional experimental work has recently been completed at the University of Frankfurt. [23] Fast neutral hydrogen and helium beams were collided with a helium target in order to provide data for benchinark testing of theory. An example of these data are shown in Fig. 8. The large peak centered near $500 \mathrm{eV}$ is due to projectile ionization since the majority of the projectile electrons are emiited with small kinetic energies in the moving projectile reference frame. Hence, in the laboratory frame, they appear as a peak centered on an electron velocity equal to the projectile velocity. The coincidence measurement (solid diamonds) confirms that the electrons in this peak are associated with ionization of the projectile. In addition the coincidence measurement indicates that a significant number of the electrons emitted with energies smaller and larger than this peak are aiso associated with projectile ionization. These electrons were originally identified by DuBois and Manson [21] as being target electrons that were emitted in simultaneous ionization events.

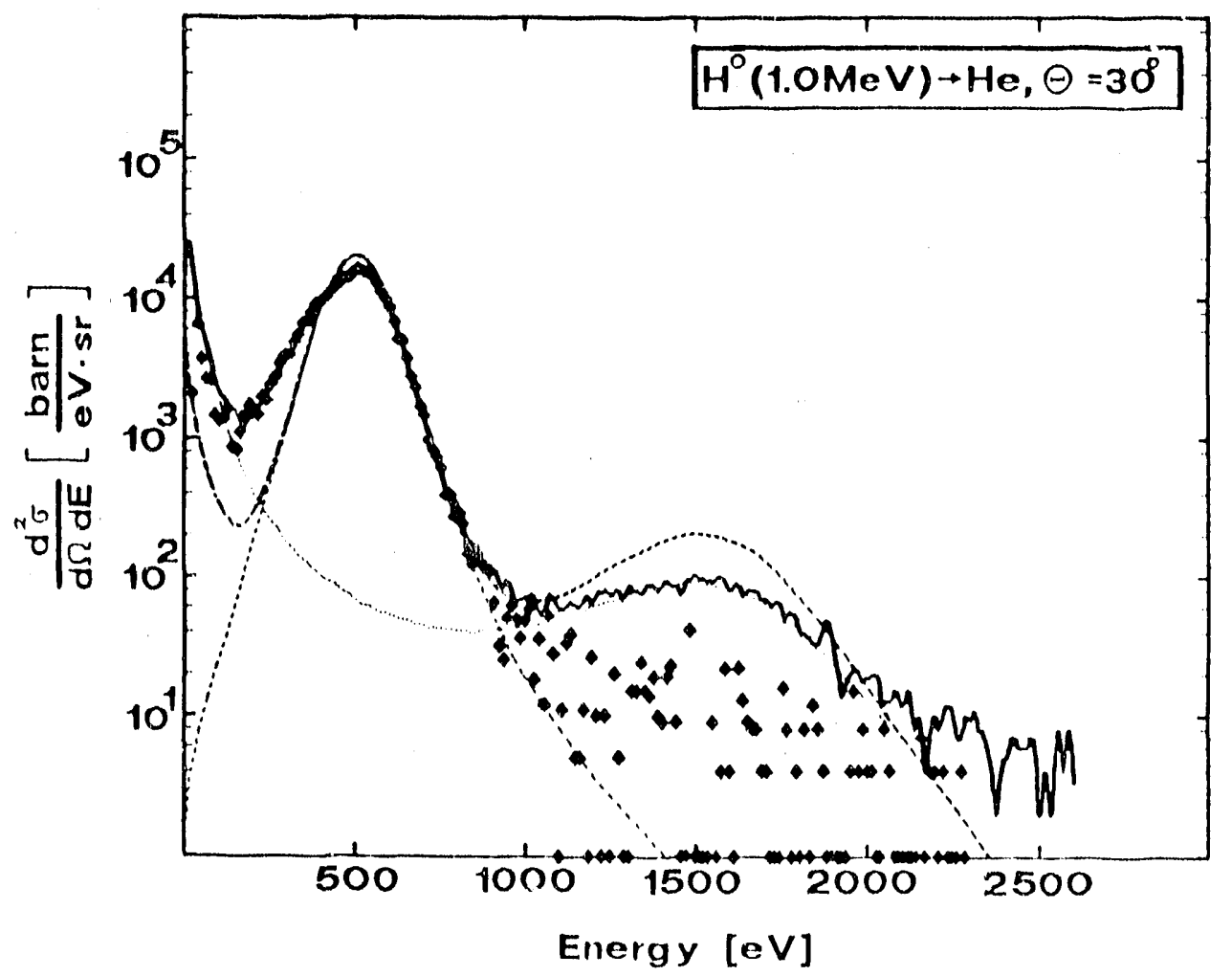

Fig. 8 DDCS for electron emission in $1 \mathrm{MeV} \mathrm{H}$ - He collisions. Experiment: solid line, total electron emission at $30^{\circ} ;$, electron-ionized projectile coincidence data. PWBA theory: -- -- --, total electron emission; - - - - projectile ionization, ...... target ionization for proton impact. Data from ref. 23 . 
The dotted and dashed curves in Fig. 8 are theoretically predictec cross sections obtained using a simple PWBA calculation. Comparisons between experiment and theory for other impact energies and angles indicated that projectile and target ionization are handled reasonabily well by the PWBA theory. Thus, it was concluded that the discrepancies previously noted for $\mathrm{He}^{+}$- Ar collisions are due to inadequate wave functions used in the calculation. Additional details and data for neutral hydrogen and helium impact can be obtained from another paper in this set of proceedings.

Coincidence measurements between ionized projectile ions and zero degree electron emission (cusp electrons) have also been studied by the Debrecen [14] and ORNL groups. [1] Data from referell $\Rightarrow 14$ are shown in Fig. 3 of this paper.

\section{c) Scattered projectile ion-sitted electron coincidences}

At the University of Frankfurt, impact faralleter measurements of the ELC process have recently been performed. [19] Electron emission at $180^{\circ}$ was investigated for $\mathrm{He}^{+}$- Ne. A constant ionization probability as a function of impact parameter was found (see Fig. 9). This is understandable since electron loss at $180^{\circ}$ results from direct collisions between the projectile electrons with the target nucleus without influencing the projectile trajectory.

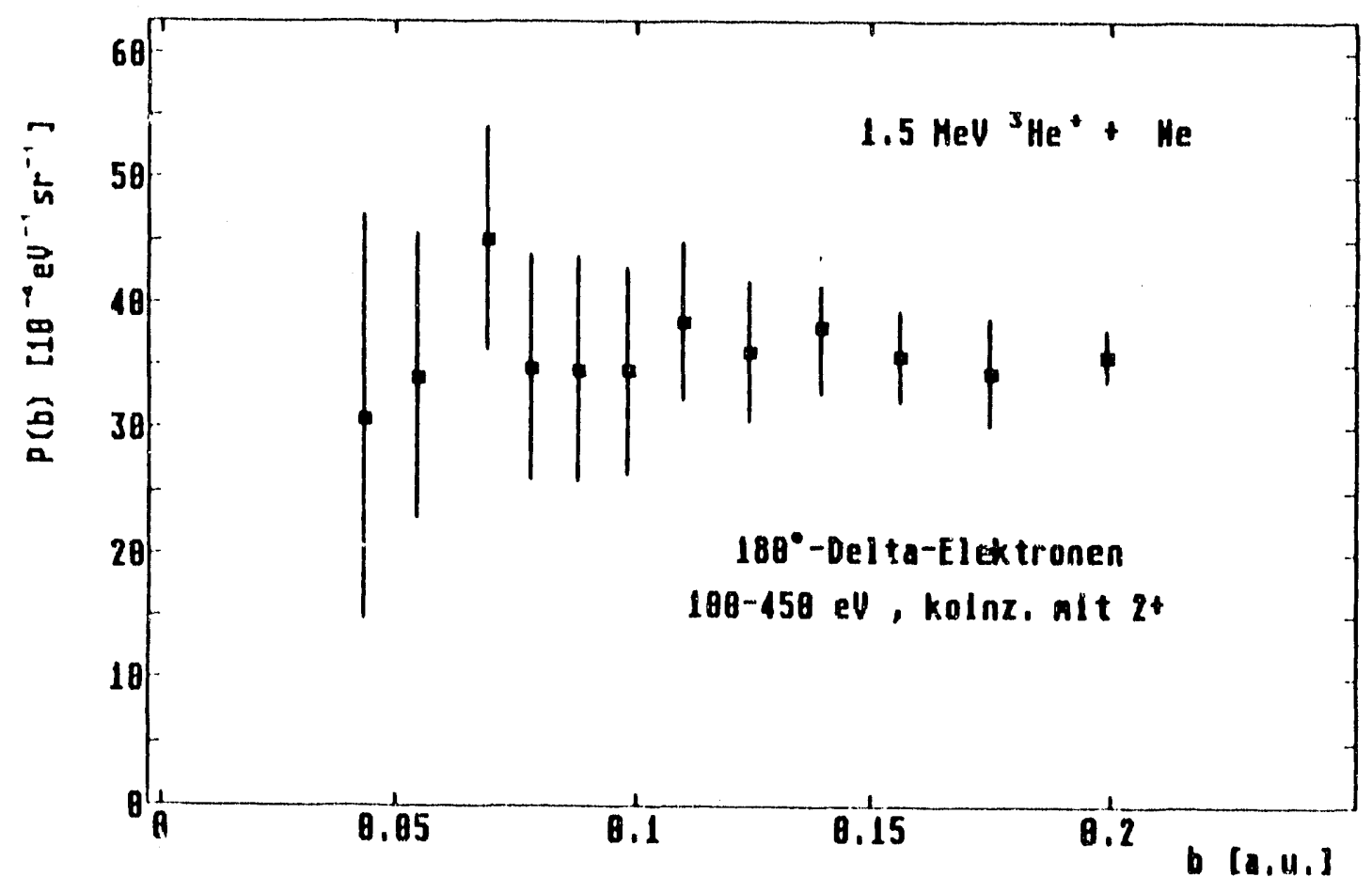

Fig. 9 The impact parameter dependence electron loss from the projectile ohserved at $180^{\circ}$ in $500 \mathrm{keV} / \mathrm{amu} \mathrm{He} \mathrm{H}^{+}$- Ne collisions. Data from ref. 19. 


\section{Conclusion:}

Although this brief survey by no means covers all invesi:gations of the electron capture and loss processes, it should provide a tlavor of the range of experimental methods being used to unraiel the mechanisms involved. Clearly the next generation of experiments will be even more sophisticated. But the studies already undertaken or in progress have provided maly new insights into these complicated processes. Ail of the examples cited are useful in providing details about these processes; however more information is obtainable from experiments where the differential electron emission or the impact parameter dependencies are measured. Hopefully additional studies of these types will be performed in the near future.

Acknowledgments: This work supported by the Office of Health arid Environmental Research (OHER), U.S. Department of Energy, under contract ifo. DE-ACO6-76RL0 1830.

\section{References:}

1. M. Breinig, S.B. Elston, S. Huldt, L. Liljeby, C.R. Vane, S.D. Berry, G.A.

Glass, M. Schauer, I.A. Sellin, G.D. Alton, S. Datz, i. Overbury, R. Laubert and M. Suter, Phys Rev A 25, 3015 (1982).

2. M.E. Rudd, R.D. DuBois, L.H. Toburen, C.A. Ratcliffe and T.V. Goffe, Phys Rev , 28, 3244 (1983).

3. M.E. Rudd, T.V. Goffe, A. It oh and R.D. DuBois, Phys Rev A 32, 829 (1985).

4. R.D. DuBois, Phys Rev A 39, 4440 (1589).

5. R.D. DuBois, Phys Rev A $\overline{3} \overline{6}, 2585$ (1987).

6. R.D. DuBois, Phy's Rev Le $\overline{t t} 52,2348$ (1984).

7. H. Damsgaard, H.K. Haugen, $\overline{\mathrm{P}}$. Hvelplund and H. Knudsen, Phys Rev A 27,112 (1983).

8. V.V. Afrosimov, Y.A. Mamaev, M.N. Panov and V. Uroshevich, Zh Tekh Fiz 37,717 (1967), [Sov Phys Tech Phys 12, 512 (1967)].

9. V.V. Afrosimov, Y.A. Mamaev, M.N. Panov and N.V. Fedorenko, Zh Tekh Fiz 39, 159 (1969), [Sov Phys Tech Phys 14, 109 (1969)].

10. M.B. Shah, P. McCallion and H.B. Gilbody, J Phys B 22, 3037 (1989).

11. L.H. Thomas, Proc Roy Soc London 114, 561 (1927).

12. R. Hippler, G. Schiwietz and J. Bossler, Phy; Rev A 35, 485 (1987).

13. J. Pálinkás, R. Schuch, H. Cederquist and 0 . Gustafsson, Phys Rev Lett 63,2464 (1989).

14. A. Kövér, L. Sarkadi, J. Pálinkás, D. Berényi, Gy Szabó, T. Vajnai, 0. Heil, K.-0. Groeneveld, J. Gibbons and I.A. Sellin, J Phys B 22, 1595 (19́89).

15. S. Datz, C. Bottcher, L.H. Andersen, P. Hvelplund and H. Knudsen, Nuil. Inst and Meth B10/11, 116 (1985).

16. T.A. Underwood, M. Breinig, C.C. Gaither III and J. Freyou, Phys Rev A 38,6138 (1988).

17. E. Horsdal, B. Jensen and K.0. Nielsen, Phys Rev Lett 57, 1414 (1986).

18. A. Skutlartz, S. Hagmann and H. Schmidt-Böcking, J Phys B 21, 3609 (1988).

19. 0. Jagutzki, Diplomarbeit (University of Frankíurt) (1989) .

20. I.S. Dmitriev, V.S. Nikolaev, L.N. Fateeva and Ya.A. Teplova, Sov Phys JETP 15,11 (1962).

21. R.D. DuBois, Phys Rev Lett 57,1130 (1986).

22. R.D. DuBois and S.T. Manson, Phys Rev A 42, 1222 (1990).

23. 0. Heil, R. Maier, M. Kuzel, K.-0. Groeneveld and R.D. DuBois, submitted to Phys Rev Lett (1990). 

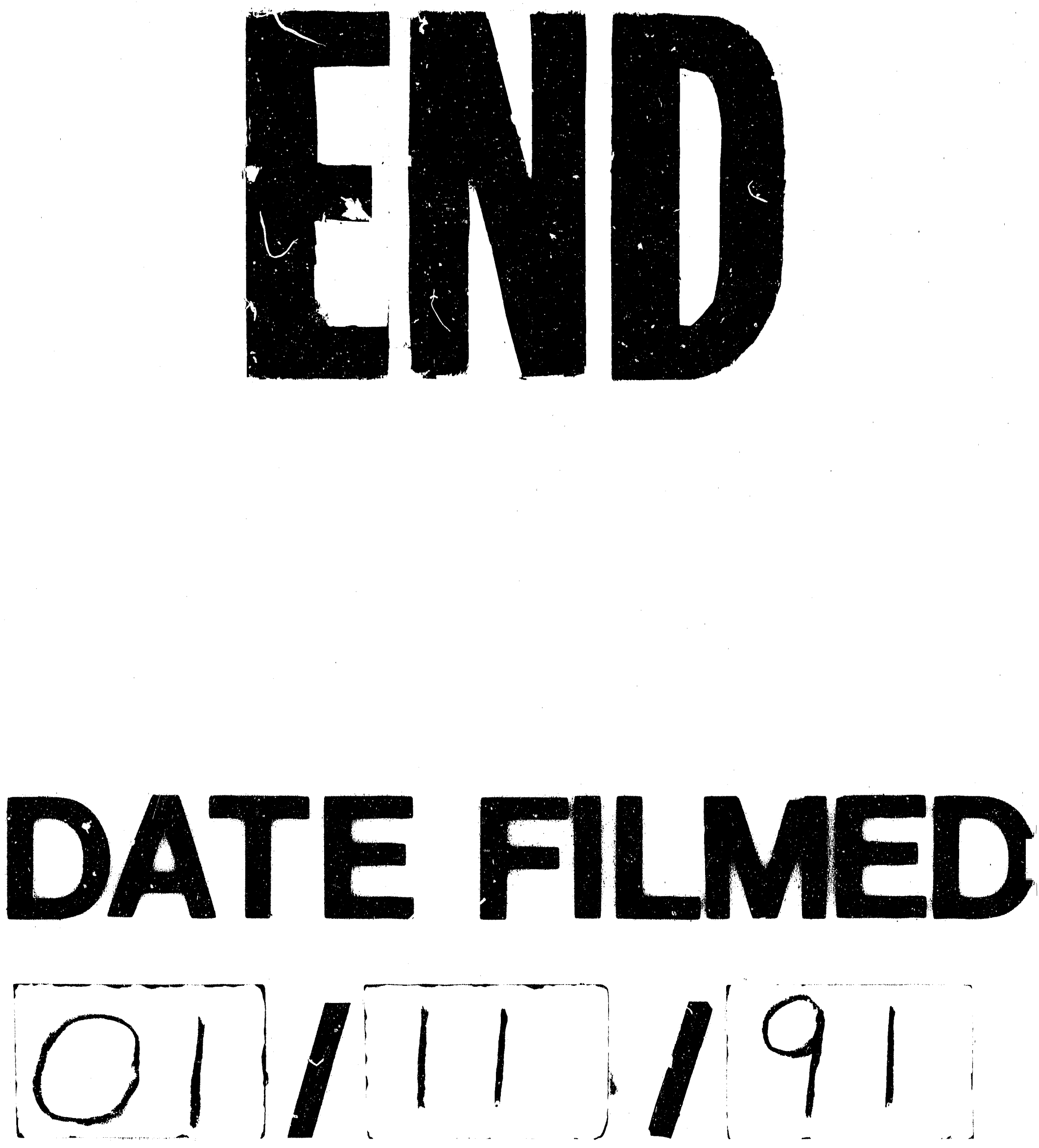
